\title{
Cross-cultural engineering skill development at an international engineering summer boot camp
}

\section{Dr. Nicholas Andres Brake, Lamar University}

Nicholas Brake is an Associate Professor in the Civil and Environmental Department at Lamar University. His research interests include engineering education, concrete pavements, fatigue and fracture of concrete material systems, the use of reclaimed materials in concrete systems, and wireless power transmission in concrete infrastructure. Dr. Brake received his Ph.D. from Michigan State University.

\section{Dr. Oleksandra Sehin, Texas State University}

Oleksandra Sehin has over ten years of experience in international and cross-cultural education. She has extensive international experience in studying, working, and living abroad. In addition to her broad education base (a Ph.D. in Adult, Professional, and Community Education, a Master's degree in Public Administration, and a combined Bachelor's and Master's degree in English language and literature), Dr. Sehin has had a comprehensive experience in the field of International Affairs related to cross-cultural advising, empathizing, and identifying with international/exchange students, faculty and scholars.

Through several years of volunteering, internship, program specialist, education abroad respresentative and education abroad coordinator positions at the International and Education Abroad offices at Texas State, she has gained experience in international/exchange student advising and programming. Oleksandra has also assisted with program evaluation reports. Currently, Dr. Sehin is employed at Texas State University in the capacity of International Affairs (Education Abroad) Coordinator.

Prior to coming to the US, Oleksandra Sehin worked as an instructor of English and Russian to the undergraduate students in a post-secondary institution of higher education in Poland for four years, and as an emigration consultant in Ukraine for two years. She is fluent in three Slavic languages - Ukrainian, Polish and Russian.

\section{Mr. John Wade Partain, Universidad Politecnica de Guanajuato \\ Dr. Damian Valles, Texas State University}

Dr. Damian Valles is a an Assistant Professor with research focused in Computer Engineering. He received his B.S., M.S. and PhD in Electrical and Computer Engineering from The University of Texas at El Paso. Dr. Valles held a post-doc position at Montana Tech in the Computer Science department as the High Performance Computing (HPC) Application Scientist. He then worked at Wake Forest University as a HPC system administrator for the Information Systems department, and part-time lecturer for the Computer Science department. Dr. Valles has been working in architecting, researching, and system administrating HPC since 2004. His research goal is to merge Engineering and Science research to HPC, Machine Learning, Embedded Systems, and Visualization solutions to enhance simulation and discovery.

\section{Dr. Alberto Marquez P.E., Lamar University}

Alberto Marquez is an associate professor in the Industrial Engineering Department at Lamar University. His previous academic appointment was Department Chair of Industrial Engineering at Tecnologico de Monterrey in Mexico City.

His research interests include modeling for decision making and optimization, with applications to supply chain, heuristic algorithm development, effective modeling and training for decision making. His publications encompass the developing of decision support systems for supply chain design; manufacturing, transportation and warehousing optimization, manufacturing capacity modeling and forecasting, simulation, scheduling, inventory policy, and process design and optimization.

He got his Ph.D in Industrial Engineering at Arizona State University in 1999 with a Fulbright-Conacyt scholarship.

\section{Dr. Jesus Alejandro Jimenez, Texas State University}


Dr. Jesus Jimenez is a Professor in the Ingram School of Engineering and the Industrial Engineering Program Coordinator at Texas State University. He received his Ph.D. in Industrial Engineering from Arizona State University. His research interests are in the modeling and analysis of manufacturing systems; computer simulation; statistical design of experiments; and sustainable production systems. His professional mission is to inspire others to improve systems through simulation modeling and analysis, applied operations research, and applied statistics.

\section{Dr. George Saltsman, Lamar University}

Dr. George Saltsman is Research Associate Professor in the Center for Doctoral Studies in Educational Leadership and currently serves as the Director of Center for Educational Innovation and Digital Learning at Lamar University. Dr. Saltsman assists UNESCO as a mobile learning policy advisor and co-chair of the Information Communication Technology thematic group for UNESCO's Teacher Task Force for Education 2030.

\section{Ms. Rosario Davis, Texas State University}




\title{
Cross-cultural engineering skill development at an international engineering summer boot camp
}

\begin{abstract}
International cross-cultural experiences have become essential components in the education of engineering students to enhance teamwork and communication skill development across different cultural and language boundaries. To this end, a customized 12-day international summer boot camp was designed to bring together third-year students from Universidad Politécnica de Guanajuato (Mexico) and post-grad students from two United States institutions (Lamar University and Texas State University) to i) promote cross-cultural collaboration and skill development in engineering, ii) increase awareness of study abroad opportunities, iii) create a network of students and faculty experts to interact and share ideas and resources in English, and iv) increase confidence in public speaking. The camp was hosted by Texas State University. The engineering students participated in several hands-on activities, that included both nontechnical skill activities in the beginning of the camp that transitioned to more technical, engineering-related activities at the end of the camp. Some of the activities included: i) contextualized grammar and vocabulary exercises, ii) contextualized team building and leadership training using Six Thinking Hats, iii) delivering technical and non-technical engineering presentations, iv) engineering design, building, and testing using CAD and 3D printing, v) design of lean assembly lines, vi) product life management, vii) discrete event simulation of an auto-part manufacturing process, and viii) using machine learning for detection of faulty automobile components. In addition to curricular camp activities, the students also participated in several fun extra-curricular activities that included a river float near campus.

Program outcomes and objectives were assessed by quantitatively capturing student crosscultural skill development, confidence, and teamwork skills using a pre/post questionnaire. The findings suggest that having engineering students from Mexico and the United States working as one team provided a unique opportunity for students to understand the importance of enhancing communication and interpersonal skills in order to be successful in solving real-world engineering problems. Student engineering teams were able to communicate effectively and evaluate work to improve design effectiveness while solving a real-world engineering design problem. Furthermore, by working in a team with culturally diverse students, participants better understood the role that a diverse group plays in the learning process in general and in personal development in specific. Participants have increased their self-confidence and improved their interpersonal skills. They acquired a better understanding of how to interact with people from different cultures and learned other ways of seeing the world.
\end{abstract}

\section{Introduction}

Cross-cultural skills are essential in today's work life and are valued necessities for organizations operating in a global environment. The ability to work effectively in international settings and connect easily with people from other backgrounds, awareness of diverse cultures, perceptions, and approaches as well as the capacity for effective communication across cultural and language boundaries - these are the skills that college graduates need to develop in order to live and work comfortably in a global society [1], [2]. Participation in global engineering programs may 
enhance a students' ability to respect other perspectives, enhance skills in quality leadership and provide preparation for leadership positions, and underscores the importance of non-technical communication skills. Successful global engineering programs provide a curriculum that teaches students about the contemporary states of the engineering profession in the foreign country and provides an opportunity to interact with engineering from other countries [3].

A pilot engineering boot camp/study abroad program-Building Bridges for New Innovators in Engineering (BBNIE), was developed to prepare an increased number of STEM graduate students for employment in today's globalized workforce. Universidad Politécnica de Guanajuato (UPG) in Cortazar, Guanajuato, Mexico, and the Texas International Education Consortium (TIEC), working with its member universities, Texas State University (TXST) and Lamar University (LU), established a study abroad partnership focused on STEM competencies and non-technical skills needed to increase the diversity of its study abroad participants as well as the diversity of the aligned academic programming in engineering. The 12-day pilot camp had participants from TXST and LU (post-grad students) and third year engineering students from UPG. Students earned college credit from their home institutions for participation in the program. The program was designed to allow UPG's STEM students to further develop their engineering skills by using the latest technology while solving real-world engineering problems they will face in the labor market. The objective was to have UPG students alongside Texas engineering students from TIEC's own TXST and LU universities. Having STEM students from Mexico and the United States learn these concepts together provided unique opportunities that advance cross-cultural cooperation and understanding. The program contained many of the elements that are known in the literature to deliver a successful experience in the development of global competency and cross-cultural skills: development of proficiency in a second language, and participation in an immersive international experience with integration of students' majors that ties various engineering elements together [4].

This paper presents an overview of the global summer engineering program which includes the curricular activities, a summary of the first-year participant demographics, and a summary of recorded pre/post survey responses serving to quantify student cross-cultural beliefs, reasons for participation, learning and expectations, and engineering teamwork effectiveness. The paper is organized with three major sections that discuss the Implementation, Methods, and Results of the program. The Implementation section discusses the pre- and camp soft-skill communication and engineering activities. The Methods section covers the cross-cultural skill assessment and demographics. Finally, the Results and Discussion section explains the analysis for reasons for participation, learning and expectations, teamwork effectiveness, and discussion. The engineering activities are described in detail in Appendix A.

The research objective is to assess the impact of the global engineering summer camp experience on students' 1) cross-cultural skill development: i) understanding the role that a diverse group plays in the learning process, and ii) understanding on how to interact with people from different cultures, and 2) self-confidence to complete both technical engineering and nontechnical work in a professional international setting. The research hypothesis is the summer camp intervention will enhance the development of students' cross-cultural skills and increase confidence in their ability to complete engineering and non-technical related tasks in an international setting. 


\section{Implementation}

\section{Pre-camp activities}

Prior to the bootcamp and arriving to the United States, UPG students were provided some technical videos pertaining to content that would be covered during the 12-day camp. This was done to ensure students had minimal competency with various engineering software programs that would be used in team settings during the camp. The UPG participants also attended a weeklong US culture seminar, led by UPG's coordinator of Languages and Internationalization to prepare students for life in the United States and university campuses.

\section{Camp activities}

The camp was divided into two parts: 1) Professional Communication Activities (1 day), and 2) Engineering Activities (5 days). The remaining days ( 6 days) were designed for travel and extracurricular activities that included the State Capitol and University of Texas engineering school tour in Austin, TX, and a river float in San Marcos, TX. The participants arrived in Austin, TX to complete the non-technical skill activities. Upon completion of the non-technical skill activities, the students then travelled to San Marcos, TX to complete the engineering activities. The students were provided room and board at the TXST dormitories. The students arrived at the sessions from the dormitories each morning, completed various activities (2-3 hours), went on a lunch break (1 hour) and returned to complete the remaining afternoon activities (2-3 hours). After completing the afternoon activities, the students returned to the dormitories and were provided dinner.

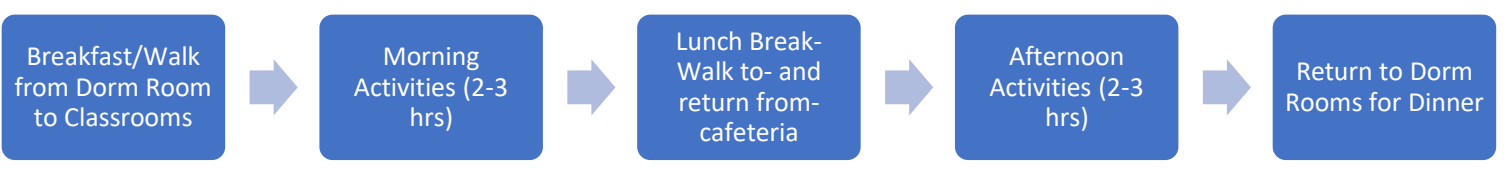

\section{Program Curriculum}

The BBNIE Professional Communication Day focused on English language features of expression and grammar that serve engineering professionals in the work environment. Activities were based on work-related situations and international events for which engineering solutions were required. Students identified and adopted useful vocabulary and grammar structures, using these to design and deliver a group presentation which addressed an international engineering concern.

- Foundations of leadership and leadership theory.

- Leadership strengths assessment and explored how strengths uniquely empower the students a leader.

- Exploration of students' native culture using Hofstede's Cultural Dimensions [5] and compared against other cultures.

- Included oral presentation regarding similarities and differences between cultures, expected business etiquette, potential team dynamics.

- Reflection of individual values as a person and robustly engaged in discussions regarding ethical challenges and how to respond to ethical challenges in the workplace. 
- Team Activity: In self-selected teams, selected a real-world issue, defined that issue as a problem, refined the problem statement through iterative analysis to propose a potential engineering solution to that problem.

- Examined the potential engineering solution in greater detail using the Six Thinking Hats [6] $(6 \mathrm{TH})$ process.

- Prepared and delivered a presentation regarding potential engineering solutions to a realworld challenge.

○ Regulations and prevention methods for earthquake-resistant housing in Mexico City.

- Solutions for resiliency in rebuilding the electrical grid in Puerto Rico (including micro-grids utilizing energy storage via pumped-storage hydroelectricity).

- Addressing water shortages in Chennai, India with evaporated sea water via largescale solar stills.

- Remediation strategies and education regarding water-borne microplastics.

- Discussed and reviewed new vocabulary and provided time for reflective journaling to assist in improved English skills.

The BBNIE Engineering activities focused on introducing manufacturing, industrial, simulation, and machine learning concepts. The activities consisted of holding lectures, hands-on activities in group and individual effort, submission of reports, and presenting group goal accomplishments to the class. Students experienced working and listening to engineering concepts in English and had opportunities to apply what they learned from the non-technical activities. The full description of each engineering activity can be found in Appendix A.

- Technical Engineering Activities

O Introduction to Engineering Design: 3D Design-Build-Test Project of structural component: Structural analysis, Testing, and Designing of a Gear and Axle assembly using a 3D printer.

○ Introduction to Design of Lean Assembly Lines: Concepts for designing lean assembly lines, including 5S, single-piece flow, and kanban systems.

- Introduction to Product Life Management and Industry 4.0: Topics and demonstrations include collaborative robots (cobots), operator 4.0, augmented and virtual reality, digital twining, among other technologies.

○ Simulation and Supply Chain: Discrete event simulation and continuous simulation of dynamic systems. Students were prompted to solve a problem that needed input from all participants.

- Machine Learning: Introduced to the concept of high-performing computing as an infrastructure evolution, machine learning, computational needs, data storage space, and processor technological designs to better fit extensive simulations and data-driven needs. 


\section{Methods}

\section{Cross-cultural skill assessment}

The purpose of this assessment is to evaluate how an international/collaborative experience has influenced cross-cultural skills for students participating in the BBNIE engineering bootcamp.

A total of 15 participants received two emails to participate in pre- and post- questionnaires on the Cross-cultural/International Experience. The pre- and post- questionnaires were designed to assess how cross-cultural skills change across time. Although several well-established general inventories exist to assess students' global perspective [7] and intercultural sensitivity [8], the assessment team chose a questionnaire that had the ability to directly assess the impact of specific camp related activities on the change in students' behavior and perceptions. The assessment tool was adapted from the International Cross-Cultural Experiential Learning Evaluation Toolkit Questionnaire, designed by the SUNY COIL Center [9], [10] and was further modified to reflect the specific goals of BBNIE project. This specific questionnaire was chosen because 1) it allows one to specifically link the impact of the cross-cultural study abroad camp activities to change in students' behavior or perception, 2) it has a relatively short completion time, and 3) offers the flexibility to adjust some of the questions to better align with the learning outcomes of the BBNIE project.

Table 1. Reasons for Participation Questionnaire

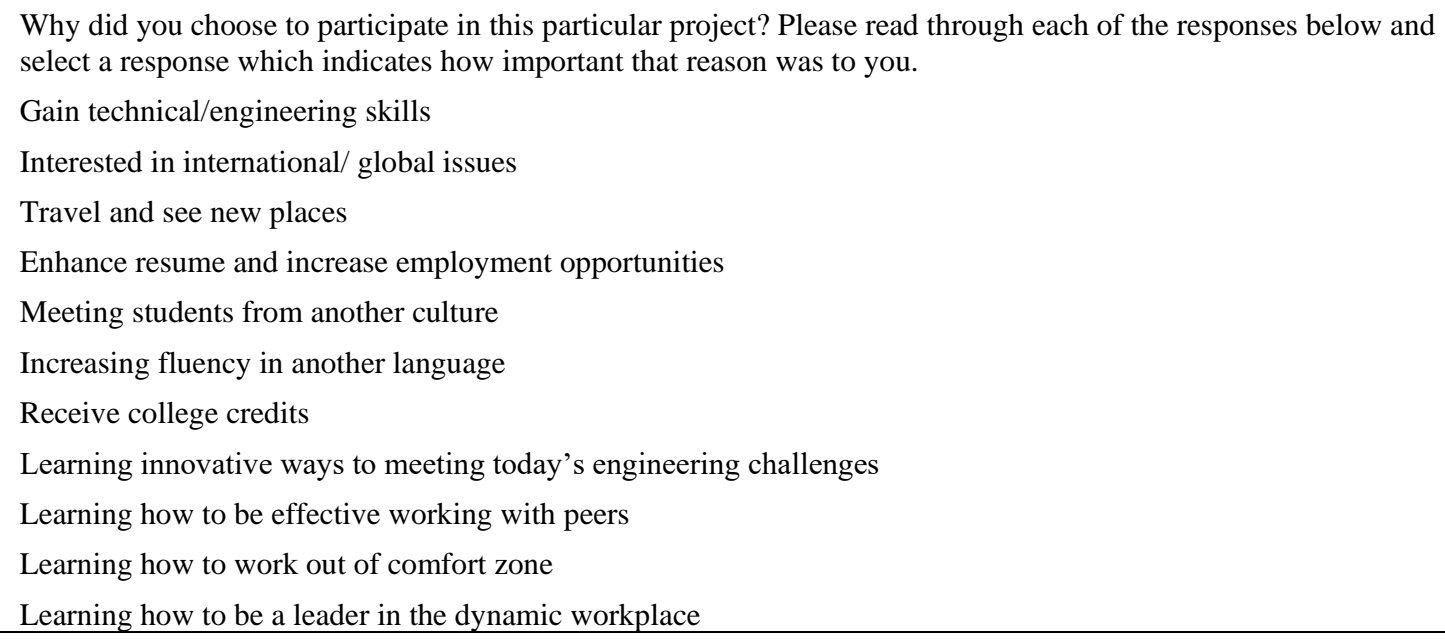

This research study was IRB approved. Pre- and post- questionnaires were entered into Qualtrics survey tool and a link to access them was sent to each student. All 15 students signed consent forms and completed two questionnaires. The questionnaires for each category are shown in Tables 1-3. The questionnaire response choices for the categories in Table 1-2 were recorded using a Likert Scale (1-4): Not/Important/Not Applicable (1), Relatively Important (2), Somewhat Important (3), and Extremely Important (4). 


\section{Table 2. Learning and Expectations Questionnaire}

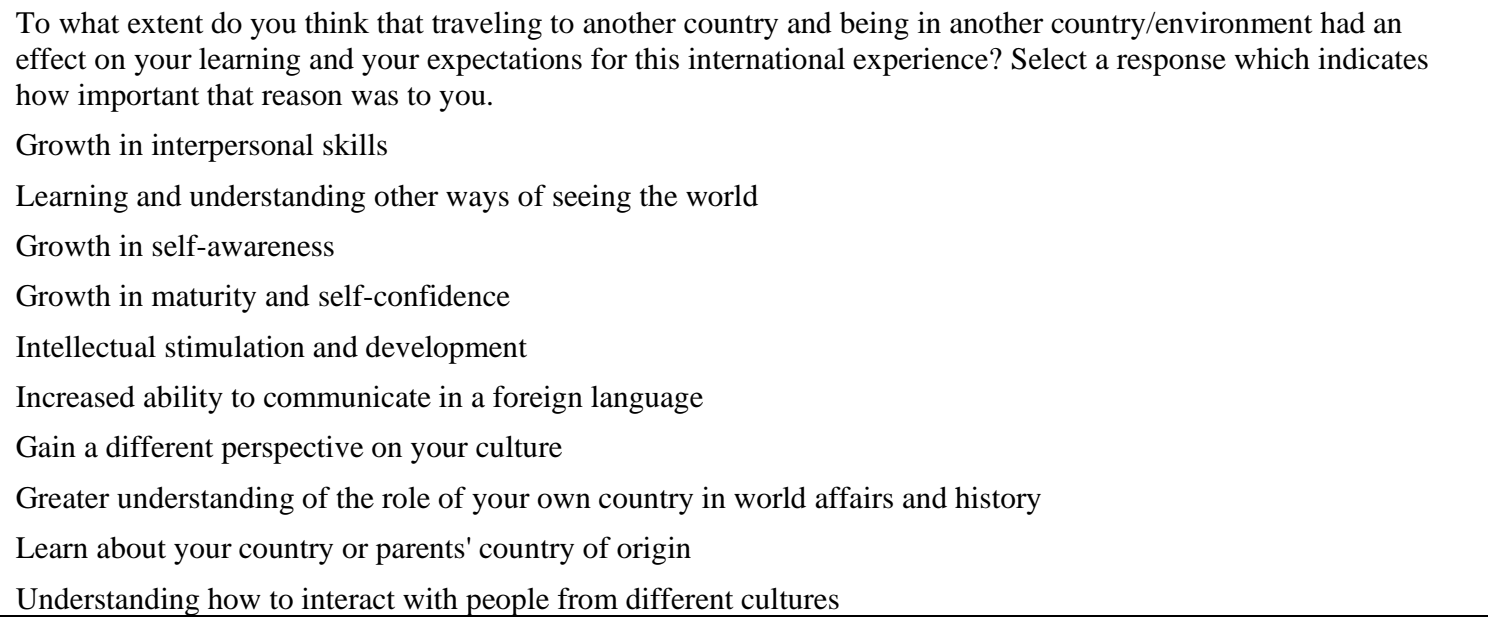

\section{Engineering team effectiveness questionnaire and assessment}

After completion of the non-technical skill training and the first engineering design team activity that involved the design a gear and axle assembly, the students were given a questionnaire to quantitatively assess team effectiveness. The questionnaire was completed in hard copy form. Each student completed the questionnaire individually and the responses were kept confidential. The study was also IRB approved and all students signed consent forms. The items on the questionnaire are shown in Table 3.

\section{Table 3. Team Member Effectiveness Questionnaire}

Select one member in your project team (not yourself) and evaluate that one person on all of the items in this survey. Strong Disagree (1) - Strongly Agree (7)

Communicated effectively

Was specific when communicating information or feelings to teammates

Expressed ideas clearly

Facilitated effective communication in the team

Helped improved communication among team members

Facilitated the exchange of ideas among team member

Provided encouragement to other team members

Was supportive of other team members.

Helped build teammates' confidence.

Used teammates' feedback to improve performance

Took teammates' feedback seriously

Acted on teammates' constructive criticism.

Assessed whether the team was making progress as expected

Checked whether the team was on the right track

Evaluated the team's work to improve team effectiveness

Fulfilled responsibilities to the team

Kept commitments to the team

Followed through on obligations to the team 
Each item was ranked on a Likert scale from 1 (Strongly Disagree) to 7 (Strongly Agree). The selected items are taken from the CATME questionnaire [11], which was validated using two studies that analyzed nearly 4,000 completed surveys. The final aggregated CATME items were validated and found to have coefficient alpha reliabilities above 0.9 .

\section{Demographics}

This section presents data on the demographic information of the participants, describes their travelling experiences prior to participation in the BBNIE project as well participants' international/cross-cultural experiences in the past. Eleven of the participants are from a Mexican Institution and the remaining four participants are studying at US institutions. Out of 15 participants, $13(87 \%)$ were male and two (13\%) female. As for the race/ethnicity, 11 participants $(73 \%)$ self-identified themselves as Hispanic, Latino or Spanish Origin, three participants (20\%) as White and one participant (7\%) as Asian/Pacific Islander (see Figure 1).

$\square$ Hispanic, Latino or Spanish origin $\quad$ White $\quad$ Asian/Pacific Islander

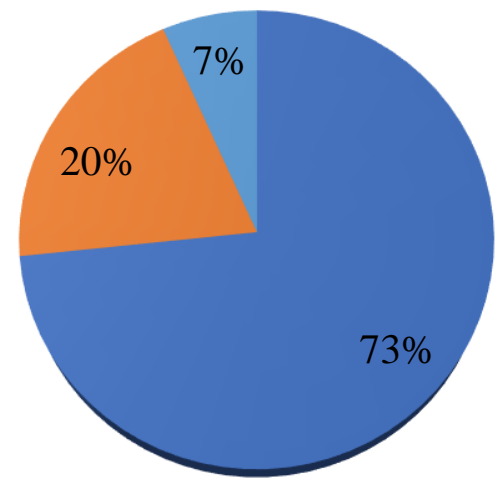

Figure 1. Race/Ethnicity

Most students, 11 participants (73\%), responded that they predominantly speak Spanish and four participants (27\%) speak other languages than English. Other languages spoken at home are Arabic, French and Hindi (see Table 4).

Table 4. Languages Spoken at Home

\begin{tabular}{lll}
\hline Only Spanish & $73.3 \%$ & 11 \\
Only English & $0.0 \%$ & 0 \\
Other languages & $26.7 \%$ & 4 \\
Total & $100.0 \%$ & 15 \\
\hline
\end{tabular}

All 15 participants travelled outside their home country prior to deciding to participate in this study abroad engineering bootcamp project. Out of 15 participants, nine $(60 \%)$ travelled outside their home country between 2-4 times, five (33\%) - more than five times and one student (7\%) indicated that she/he had travelled abroad one time prior joining this international project (see Figure 2). 

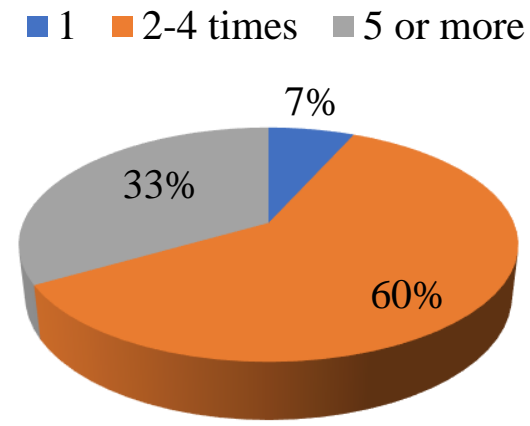

Figure 2. International Travel

The average duration of these international trips was more than two weeks according to eight participants (53\%). Three participants (20\%) spent up to one month abroad while four students (27\%) spent over one month being outside their home country (see Figure 3).

-1-2 weeks $\square 2$ weeks - 1 month $\square$ Over a month

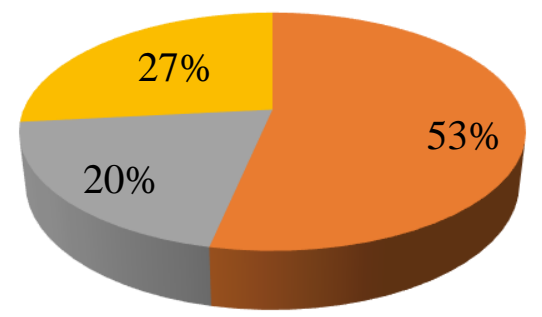

Figure 3. Duration of International Trips

All 15 participants have been involved in some form of international/cross cultural activity in the past. Out of 15 participants, five (25\%) had been involved in an internationally focused group/research at their home university or in a community and had visited relatives who reside outside the home country. Three participants (15\%) indicated that they studied abroad through a high school exchange program. Two participants (10\%) participated in an international servicelearning project and an international conference. One student indicated that he/she had not participated in an internship abroad, research abroad or short-term faculty-led program abroad. None of the participants participated in a semester or a yearlong exchange program (see Figure 4). 


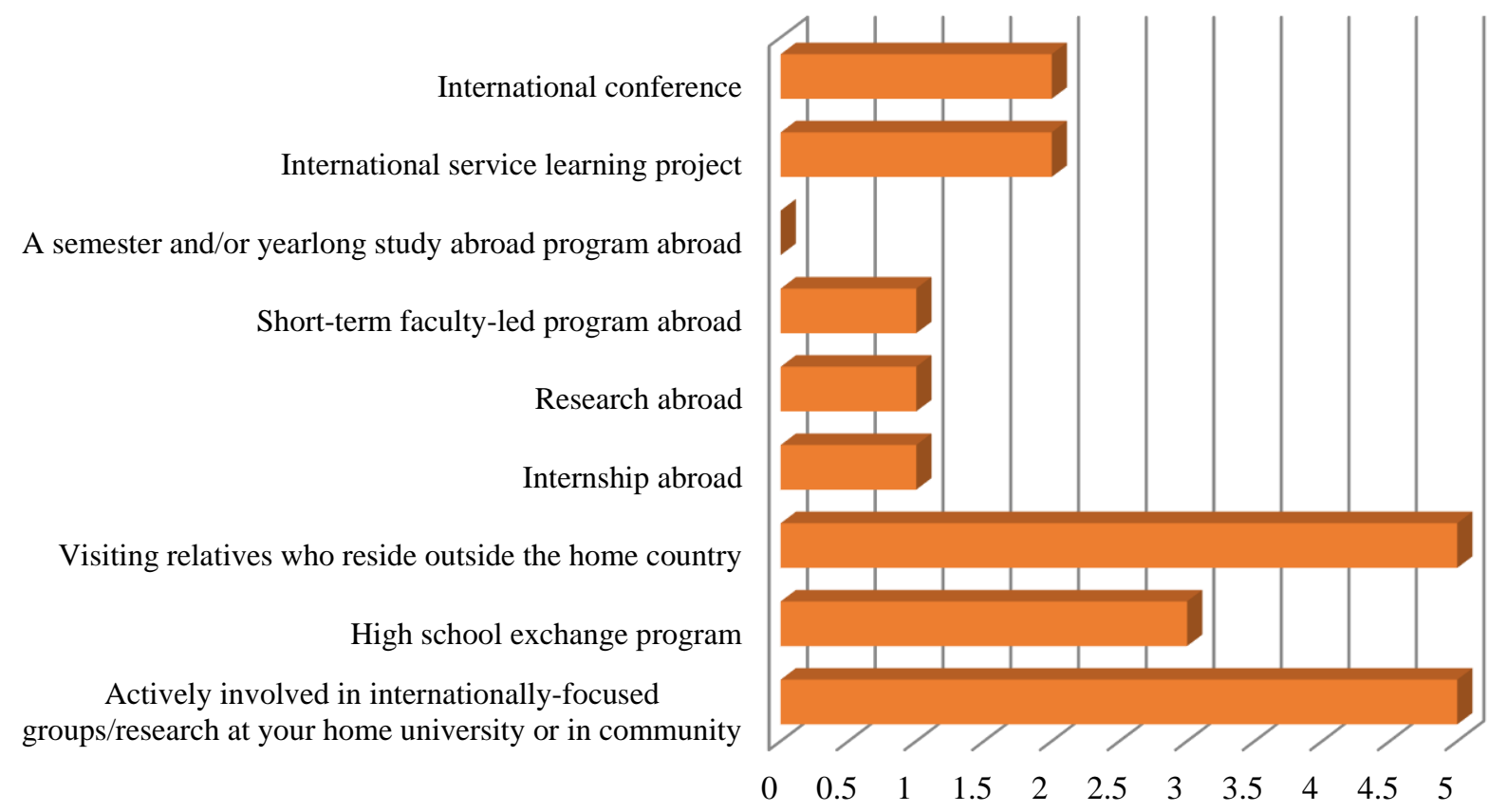

Figure 4. Number of Responses: Experience in international/cross-cultural activities

\section{Results and Discussion}

\section{Reasons for Participation}

Figure 5 shows the comparison of data from pre- and post- questionnaires as it relates to the reasons why students have decided to participate in the BBNIE project. The data shows that there was an increase from pre- to post- questionnaires as it relates to the 'extremely important' reasons in six categories and four of these categories belong to cross-cultural skills such as (1) learning how to be effective working with peers, (2) interested in international/global issues, (3) travels and see new places, and (4) increasing fluency in another language. The biggest differences in numbers were related to cross-cultural and non-technical skills, (2) receiving college skills, (3) learning how to be effective working with peers and (4) learning innovative ways to meeting today's engineering challenges. This finding suggests that after working in a team with culturally diverse students, participants better understood the role that a diverse group plays in the learning process in general and in personal development in specific. This leads to the conclusion that by participating in the engineering bootcamp, students anticipated gaining technical skills and expanding their knowledge to cross-cultural, linguistic, and non-technical skills to be well prepared for today's competitive engineering workforce. 


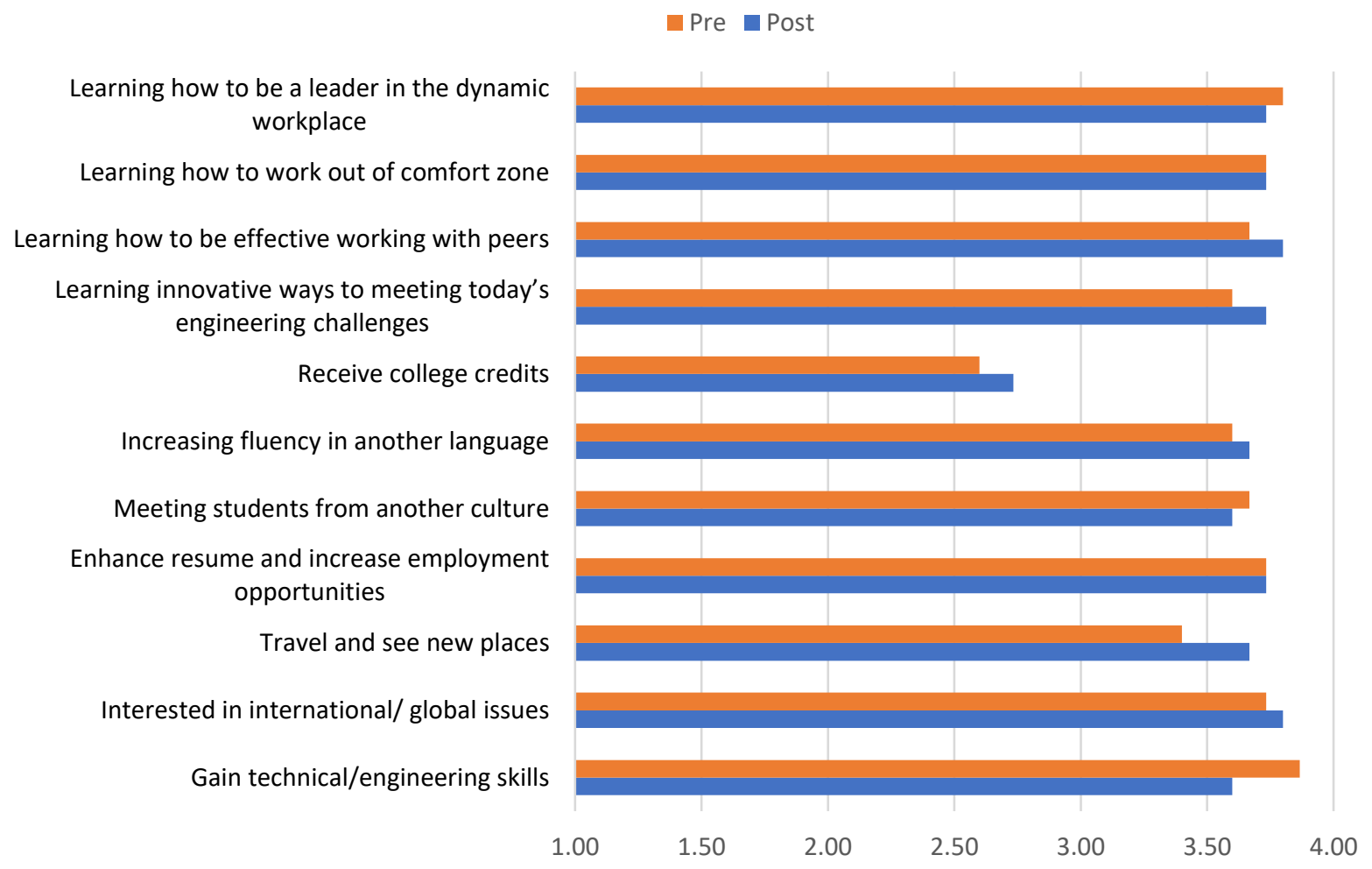

Figure 5. Number of Pre/Post-Responses for Reasons for Participation

In contrast to an increase of interest in improving cross-cultural and non-technical skills, the data also shows a slight change from 'extremely important' reasons and 'somewhat important' reasons in two categories related to workplace learning, specifically: (1) gaining technical/engineering skills and (2) learning how to be a leader in the dynamic workplace. This suggests that by participating in the bootcamp and working together with cross-cultural team members in assigned projects, some students recognized that success of the projects depends not only on the technical skills but also on how well they communicate their ideas to other colleagues and how cooperative they are in accepting other opinions as well.

\section{Learning and Expectations}

Figure 6 shows that among the expectations that were 'extremely important' for participants before joining this project and 'have had the great effect' on them after the project was over were the following: (1) growth in maturity and self-confidence and (2) understanding how to interact with people from different cultures. 


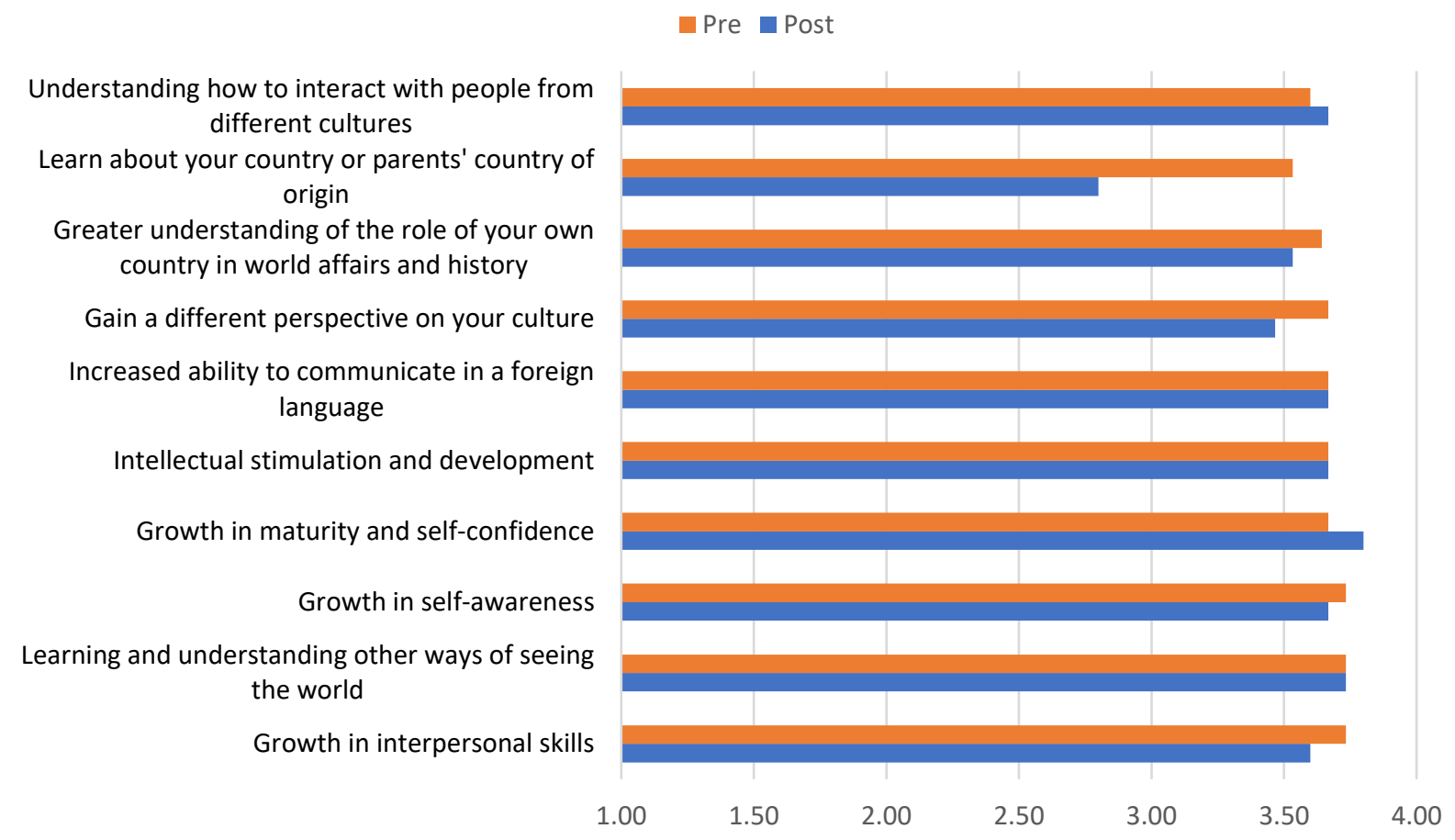

Figure 6. Number of Pre/Post-Responses for Learning and Expectations

There was a decrease in two categories: (1) growth in interpersonal skills and (2) learning about student's country or parents' country of origin. One of the factors that might have impacted this slight change is that domestic students were part of this project and, thus, were not completely exposed to a foreign country environment.

Three categories remain the same in pre- and post- questionnaires: (1) learning and understanding other ways of seeing the world, (2) intellectual stimulation and development, and (3) increased ability to communicate in a foreign language. This data suggests that most students came to this project with high expectations for learning and development of their cultural, global, and foreign language skills and these expectations were met by collaborating with other students and faculty during the engineering bootcamp project. Most expectations were rated very highly as 'somewhat important'/ 'had some effect' (between 3-6 responses) and 'extremely important' or 'had great effect on students' (between 5-12 responses). This indicates that participants are aware of the demand of today's workforce and by participating in this project they expected to gain engineering, non-technical and cross-cultural skills to be competitive in a globalized workforce.

\section{Teamwork Effectiveness}

The results of the team effectiveness survey are shown in Figure 7. The survey was given to the students immediately after completing the engineering team activity involving the design, build, test and presentation of the vehicle drive shaft and wheel assembly project (Introduction to Engineering Design Module). 


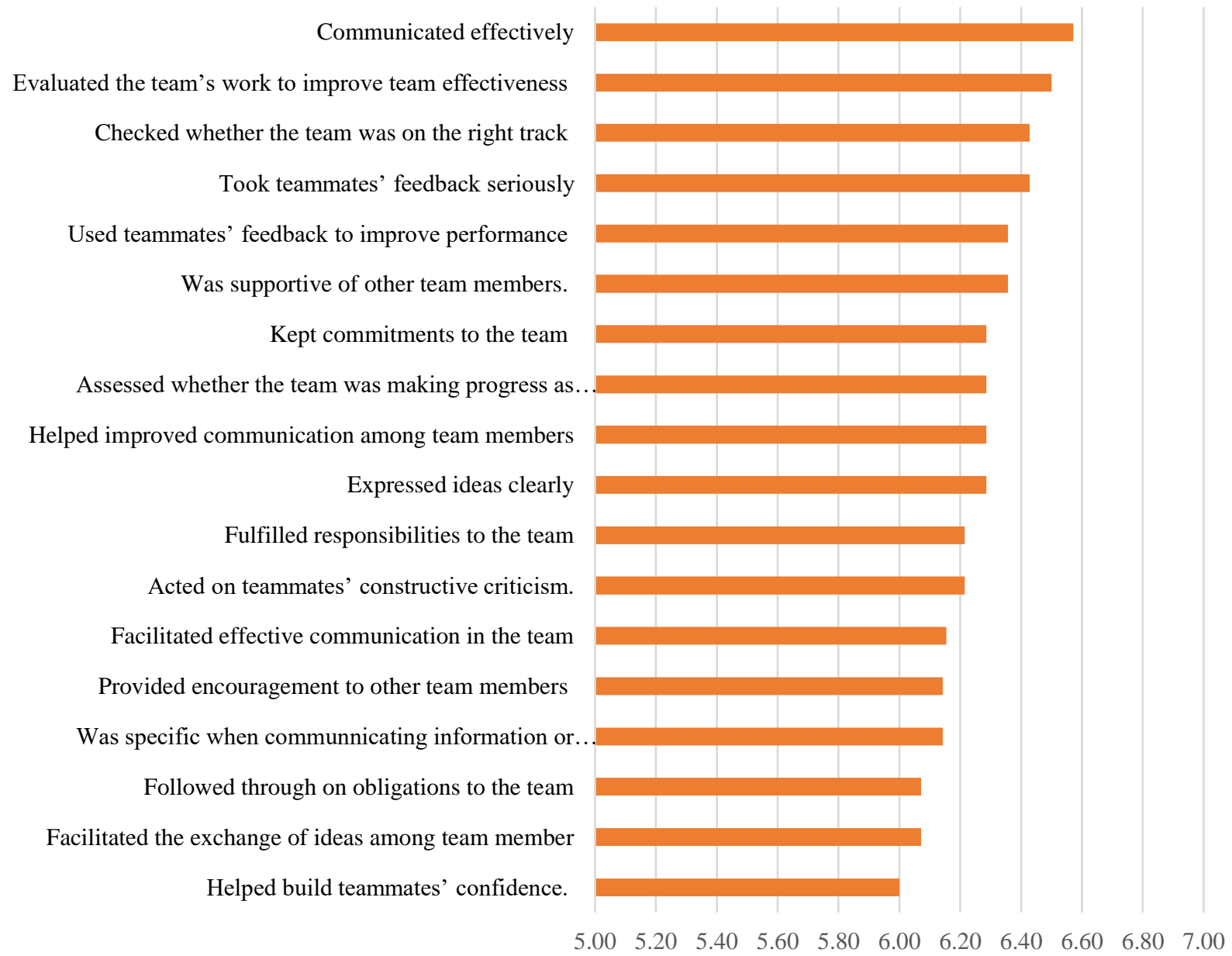

Figure 7. Results from Team Effectiveness Survey

The bar graph represents the mean response of each item. The mean data is represented in descending order for organization. The students rated their teammates' ability to communicate effectively and improve team effectiveness the highest. The lowest ranked item was helping build teammates confidence, although all the mean responses were above 6/7 (near strong agreement). This would suggest the teams were actively participating in the design process to improve and/or optimize the components and sub-components of the engineering system; allowing ideas to flow back and forth between sub-groups to ensure all engineering constraints were simultaneously being satisfied. In only 5-6 hours of working together as a team (plus the one day of non-technical skill development), the students showed they operated effectively in a team setting and were able to properly communicate both technically and non-technically. The students were able to successfully design, build, test, and present a final product design requiring the satisfaction of some realistic engineering constraints in a foreign language.

\section{Discussion}

This BBNIE project focused on cross-border education with the purpose of expanding access to cross-cultural, linguistic, and non-technical skills demanded in the engineering field on both sides of the Mexico-U.S. border. The assessment of the cross-cultural skills showed that even 
though students' initial reasons to participate in the BBNIE project were mostly related to gaining technical skills, after spending some time with team members from diverse backgrounds, students slightly shifted their focus on improving their cross-cultural and non-technical skills. The findings suggest that having engineering students from Mexico and America working as one team provided a unique opportunity for students to understand the importance of enhancing communication and interpersonal skills in order to be successful in solving real-world engineering problems. Furthermore, by working in a team with culturally diverse students, participants better understood the role that a diverse group plays in the learning process in general and in personal development in specific. Students were able to successfully communicate in English to complete a technical engineering task, were actively evaluating the team's work to improve team effectiveness, took teammates' feedback seriously, and were found to be supportive of other team members.

The findings showed that majority of participants have come to the BBNIE project with high expectations for learning and development of their cross-cultural, linguistic, technical and nontechnical skills and these expectations were met by collaborating with diverse group of students and faculty during a 12-day engineering bootcamp project. Furthermore, by participating in this short-term study abroad project, participants have increased their self-confidence and improved their interpersonal skills. They acquired a better understanding of how to interact with people from different cultures and learned other ways of seeing the world. These cross-cultural skills are the foundation for developing intercultural/global competence [12].

The findings also indicate that participants have been actively involved in international activities prior to joining this project and are eager to continue exploring the world around them and beyond. They are aware that in order to be better prepared for the global workforce they need to develop an appreciation of other cultures, respect other students' ideas, continue learning how to interact well with people and understand that they hold different interests, values and/or beliefs. Participants did acknowledge that culturally diverse teams bring unique and distinct solutions to the issues on the project they are engaged in and, thus, lead to the successful completion of the project in general. This finding is in coherence with research that greater collaboration between international and domestic students often leads to greater mutual learning [13].

\section{Conclusion}

International and study abroad programs play a significant role in developing cross-cultural, linguistic, technical, and non-technical skills for an ever-changing globalized workplace. Crosscultural skills or global competence can be achieved by redesigning curriculum to reflect international components, by extending foreign language preparation of students, by offering cross-cultural international projects, etc. Since global competence is among the new skills needed for all students to be successful in a global society, universities should provide more opportunities for students to participate in study abroad/international projects.

\section{Acknowledgements}

The BBNIE project was funded by ExxonMobil in conjunction with the 100,000 Strong in the Americas and Partners of the Americas. The views, thoughts, and opinions expressed in this paper belong solely to the authors, and do not necessarily reflect the views of the funding agencies. 


\section{References}

[1] W. I. Brustein, "The global campus: Challenges and opportunities for higher education in North America,” J. Stud. Int. Educ., vol. 11, no. 3-4, pp. 382-391, 2007.

[2] M. F. Green, "Measuring and assessing internationalization," NAFSA Assoc. Int. Educ., vol. 1, pp. 1-26, 2012.

[3] G. L. Downey et al., "The globally competent engineer: Working effectively with people who define problems differently," J. Eng. Educ., vol. 95, no. 2, pp. 107-122, 2006.

[4] J. R. Lohmann, H. A. Rollins, and J. Joseph Hoey, "Defining, developing and assessing global competence in engineers,” Eur. J. Eng. Educ., vol. 31, no. 1, pp. 119-131, 2006.

[5] G. Hofstede, "Geert Hofstede cultural dimensions," 2009.

[6] E. De Bono, Six thinking hats. Penguin UK, 2017.

[7] L. A. Braskamp, D. C. Braskamp, K. C. Merrill, and M. Engberg, "Global Perspective Inventory (GPI): Its purpose, construction, potential uses, and psychometric characteristics," Retrieved January, vol. 12, p. 2010, 2008.

[8] M. R. Hammer, M. J. Bennett, and R. Wiseman, "Measuring intercultural sensitivity: The intercultural development inventory," Int. J. Intercult. relations, vol. 27, no. 4, pp. 421-443, 2003.

[9] S. Guth, "The COIL Institute for Globally Networked Learning in the Humanities," Final report. New York, NY SUNY COIL Cent., 2013.

[10] B. Chandra, K. Landa, R. Smolar, R. Mukherji, P. P. Torcivia, and S. Jagendorf-Sobierajski, "Cross-Cultural Experiential Learning Evaluation Project," 2013.

[11] M. L. Loughry, M. W. Ohland, and D. DeWayne Moore, "Development of a theory-based assessment of team member effectiveness," Educ. Psychol. Meas., vol. 67, no. 3, pp. 505$524,2007$.

[12] B. Leask, "Using formal and informal curricula to improve interactions between home and international students," J. Stud. Int. Educ., vol. 13, no. 2, pp. 205-221, 2009.

[13] O. V Kostareva, "US American Students' Experience with and Orientation Toward International Diversity in a Midwestern Comprehensive University Setting." University of Northern Iowa, 2006. 


\section{Appendix A: Summary of Bootcamp Activities}

\section{Engineering Activities}

\section{A.1 Engineering Design Activity, Design-Build-Test: 3D CAD + Simulation + 3D Printing}

The purpose of the activity was to place the students in a competitive team environment where both non-technical and technical communication is needed between participating team members. Additionally, this exercise directly introduces students to engineering design, 3D CAD, 3D structural simulation, and 3D printing. The students were required to design, build, and test a 3D printed component that was installed onto a small-scale vehicle. The students were required to test the vehicle after designing the sub-components and were required to summarize the design in a 5-minute presentation.
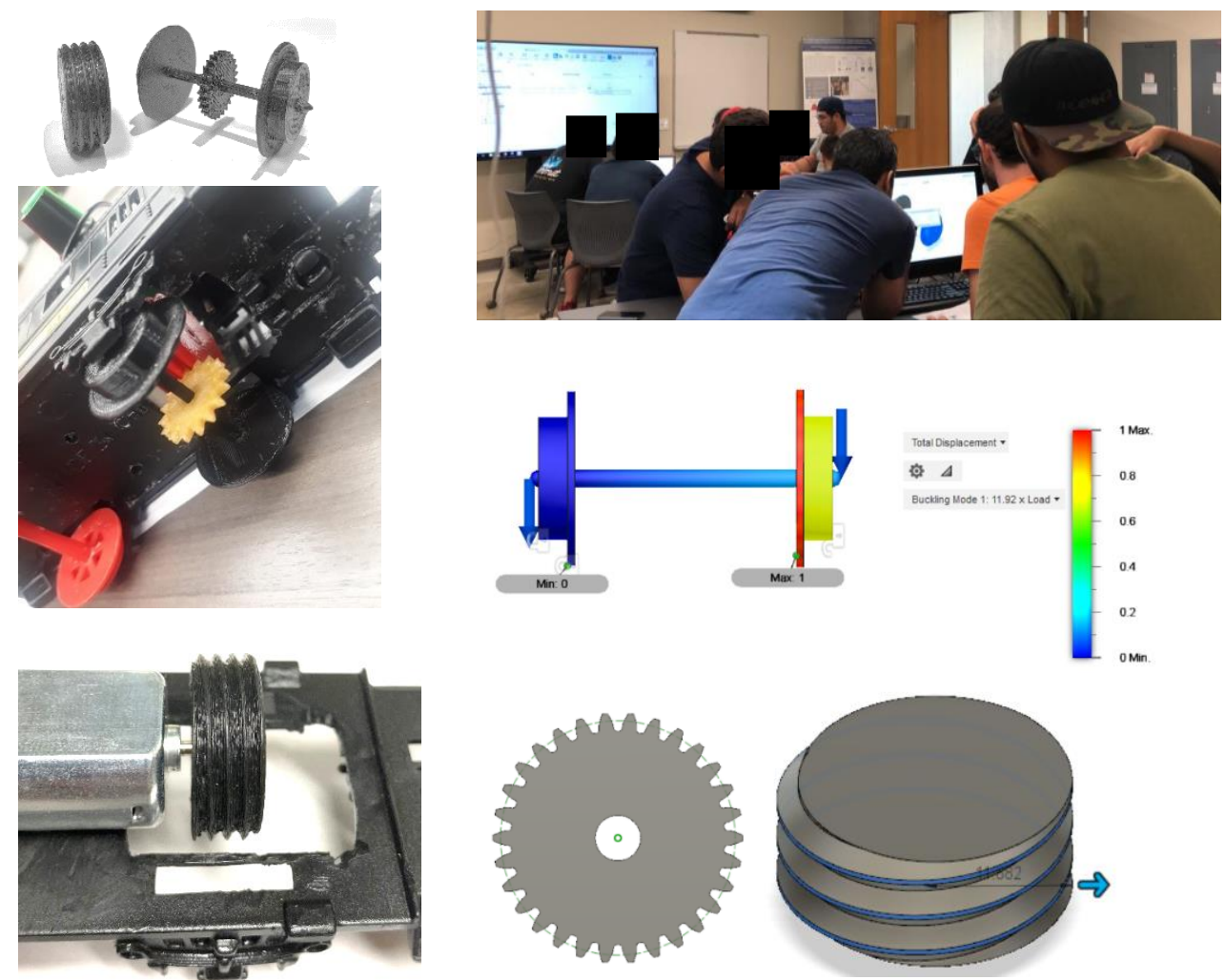

Figure A.1. Photographs/Images of Engineering Activity 1

Students were placed into two teams that competed against each other and tasked with designing different vehicle components: 1) a geared/threaded drive shaft and 2) a wheel assembly that would i) maximize travelling velocity of a fully assembled vehicle (along a designated track path), and ii) minimize the weight of the wheel assembly. Each team had six members and each team was divided into two different sub-teams: i) Drive Shaft Team and ii) Wheel Assembly Team. Each member had a defined role within each sub-team (Drive Shaft: Field Engineer, Modeler, Team Manager; Wheel Assembly: Designer, Modeler, Team Manager). Each team needed to record field data to facilitate the selection of the optimal drive shaft and were also 
required to conduct a structural static analysis and buckling analysis using 3D structural simulation software. Upon completing the design, the students were asked to 3D print the optimized vehicle sub-components, install the sub-components onto an existing vehicle system and test the vehicle velocity using a stopwatch. The Team Manager collected photographs and data from each of the sub-teams to construct the summary presentation (see Figure A.1).

\section{A.2 Introduction to Design of Lean Assembly Lines: A Simulation Game}

Concepts for designing lean assembly lines include 5S, single-piece flow, and kanban systems. These concepts are illustrated by using a lean simulation game. The participants design a multistation assembly line for building Lego cars. The Lego assembly line design experience consists of six different rounds. In the first round, the participants create an initial assembly line design and run a physical simulation to evaluate its key performance indicators, such as cycle time, throughput, and work-in process. Each participant performs a critical role during the simulation game, which includes the record keeper, the manufacturer operator, and the material handler. In subsequent rounds, the participants apply important lean manufacturing principles to improve overall system performance. The objective of the simulation game is to learn and apply basic lean manufacturing principles such as $5 \mathrm{~S}$, single-piece flow, and visual kanban systems to reduce waste from assembly operations. At the end of the simulation game, each participant presents one PowerPoint slide with a summary of activities and key lessons learned during the simulation game. Each presentation should last no more than two-minutes (see Figure A.2).

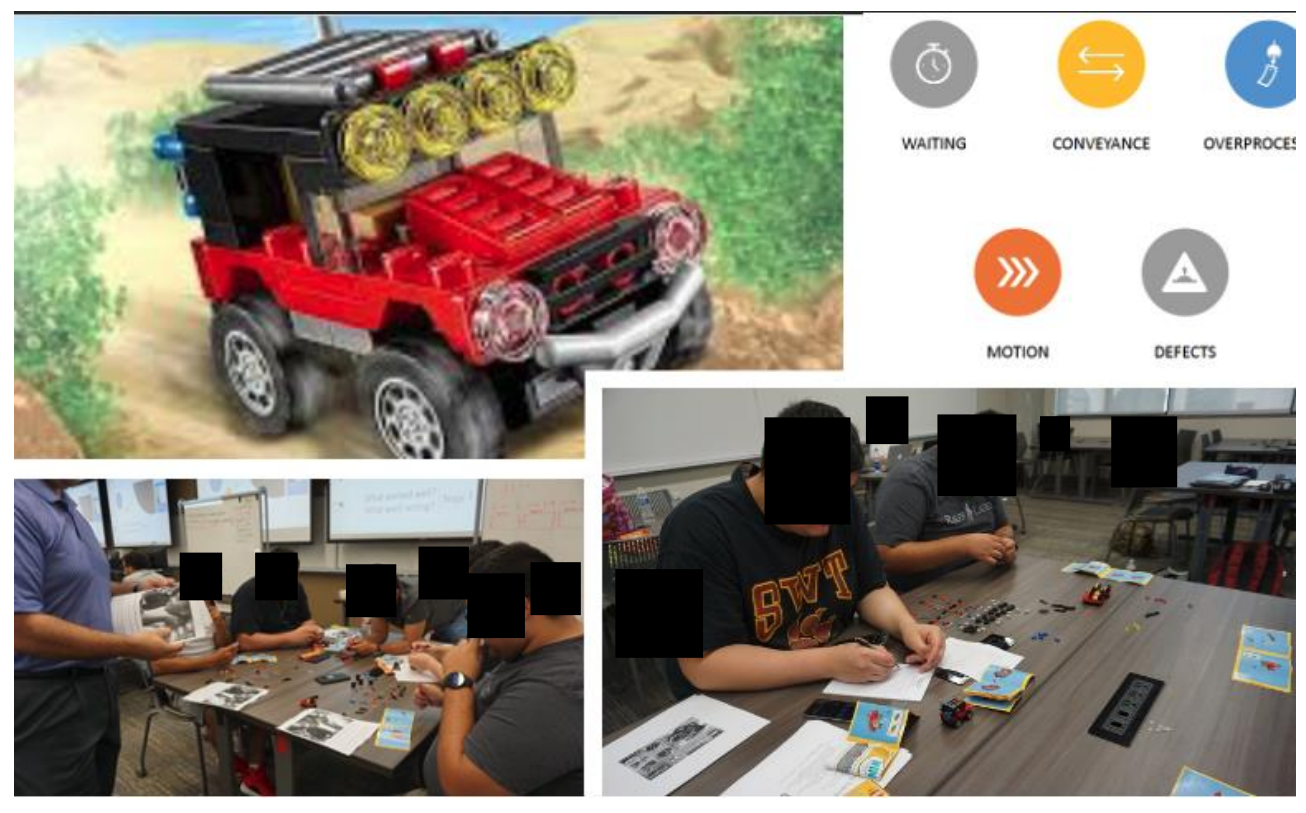

Figure A.2. Photographs of Engineering Activity 2

\section{A.3 Introduction to Product Life Management and Industry 4.0}

This series of lectures and demonstrations introduces students to the latest advancements of industry 4.0. It demonstrates different technologies used in industrial facilities and warehouses. Topics and demonstrations include collaborative robots (cobots), operator 4.0, augmented and virtual reality, digital twining, among other technologies. Special consideration is given to the penetration of renewable energy into high-tech manufacturing facilities to reduce carbon 
consumption in the industrial sector. At the end of the lecture and demonstrations, each participant presents one PowerPoint slide with a summary of activities and key lessons learned during the day. Each presentation lasts no more than two-minutes (see Figure A.3).

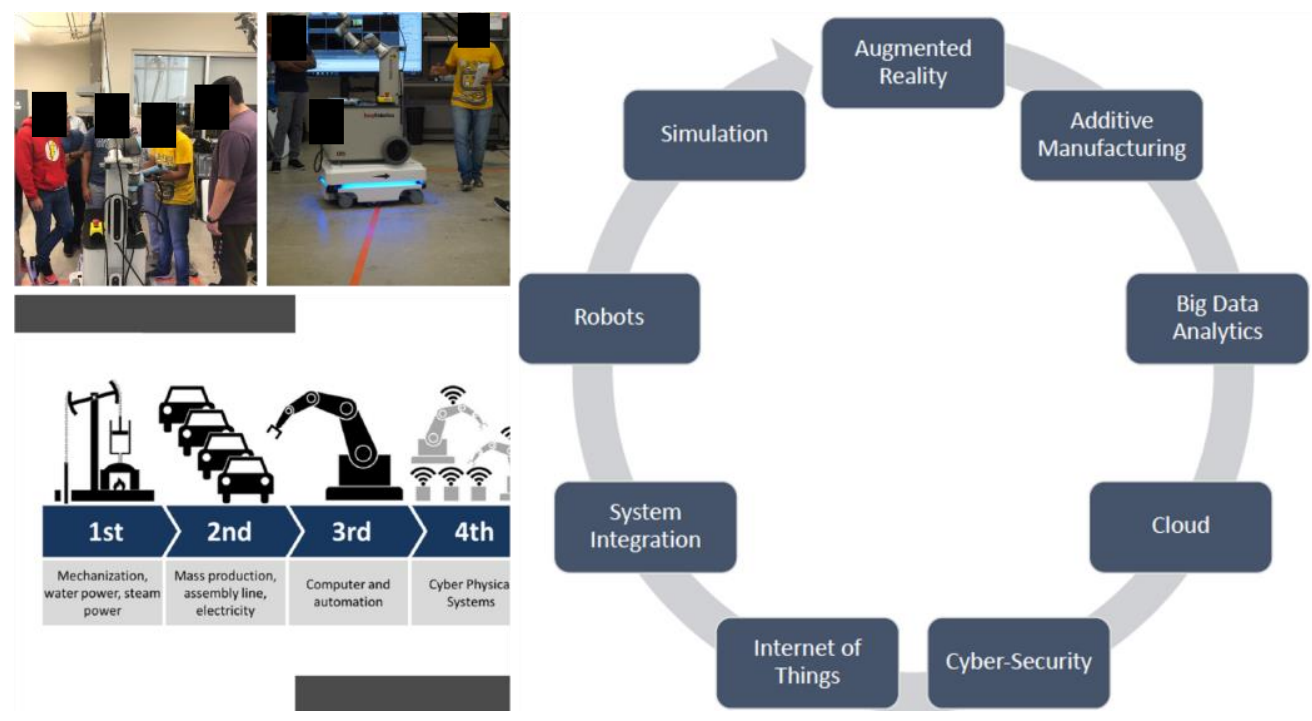

Figure A.3. Photographs of Engineering Activity 3

\section{A.4 Simulation and Supply Chain}

The main goal of the session was for students to practice their communication and leadership skills while tackling a complex technical challenge. Students were presented with two approaches to simulation of systems: Discrete event simulation and continuous simulation of dynamic systems. After a primer explanation of the capabilities of the simulation software pertaining to supply chain, students were separated into teams of 3 students. Each member of the team was given partial information for them to solve a problem that needed input from all students. Communication was allowed within groups but not between groups. For the interaction within teams, students were encouraged to use their recently practiced communication skills to be able to come to solutions where each member had partial information.

The technical challenges presented were related to auto-part manufacturing to maintain the theme of the other sessions. The activity included modeling five operations: cutting, subassembly, final assembly, inspection, and packaging. Questions included the computation of the manufacturing capacity, the utilization of resources and a comparison of push vs. pull manufacturing. Students were requested to model the use of Internet-of-Things coordination to achieve a pull- as opposed to push- manufacturing system. After concluding the discrete even simulation tasks, the students worked on a step-by-step tutorial for continuous simulation of dynamic systems for them to practice their communication skills. A short final reflection on the interaction within teams and how that was related to their communication effectiveness and technical performance concluded the session (see Figure A.4). 

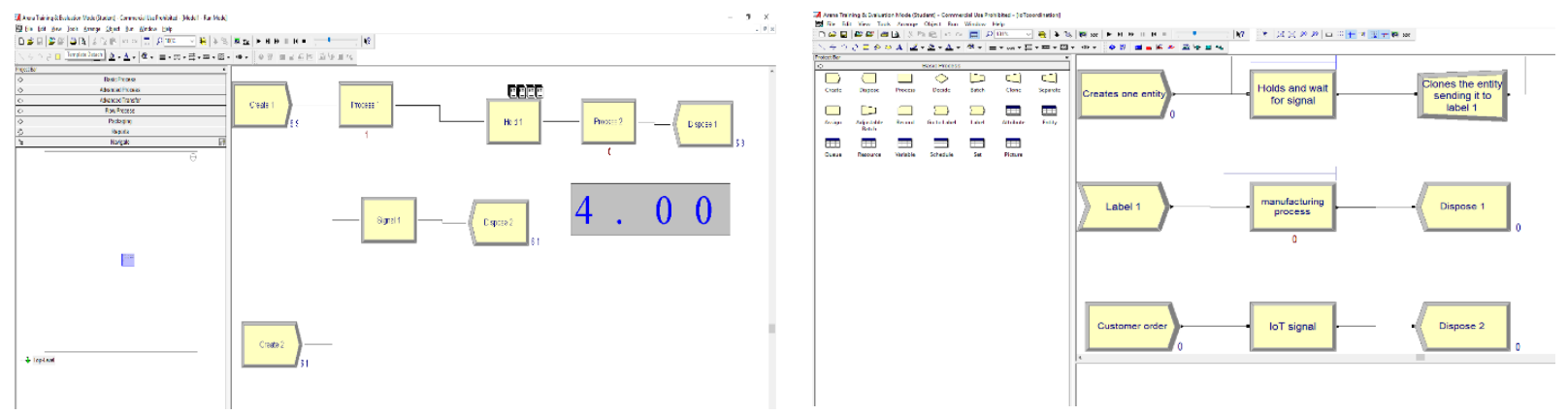

Figure A.4. Photographs of Engineering Activity 4

\section{A.5 Machine Learning}

The students were introduced to the concept of high-performing computing as an infrastructure evolution, machine learning computational needs, data storage space, and processor technological designs to better fit extensive simulations and data-driven needs. Engineering concepts were discussed in relation to how simulation and collection of data in engineering environments have become a more significant need for engineers to utilize. Then, machine learning concepts and techniques were discussed that help data engineers select the best solutions to different problems. The discussion was focused on Support Vector Machine (SVM) for classification purposes and Artificial Neural Networks (ANN) for regression modeling. Videos were then shown as different ANN models have been used for engineering applications in different industries. The session ended with a discussion of Python, Numpy, Scipy libraries that complement machine learning libraries such as TensorFlow and Keras, and parameter finetuning competition exercise. The exercise was to use an already developed ANN regression model with TensorFlow and Keras for different features that dealt with crankshaft data and outcomes. The goal was to fine-tune the parameters of the machine learning model to improve the performance of the regression behavior in classifying faulty error crankshaft standardized codes. Students were given access to a high-end server with GPU-technology to run the ANN model for the competition. The winner took home a donated Amazon Alexa (see Figure A.5).
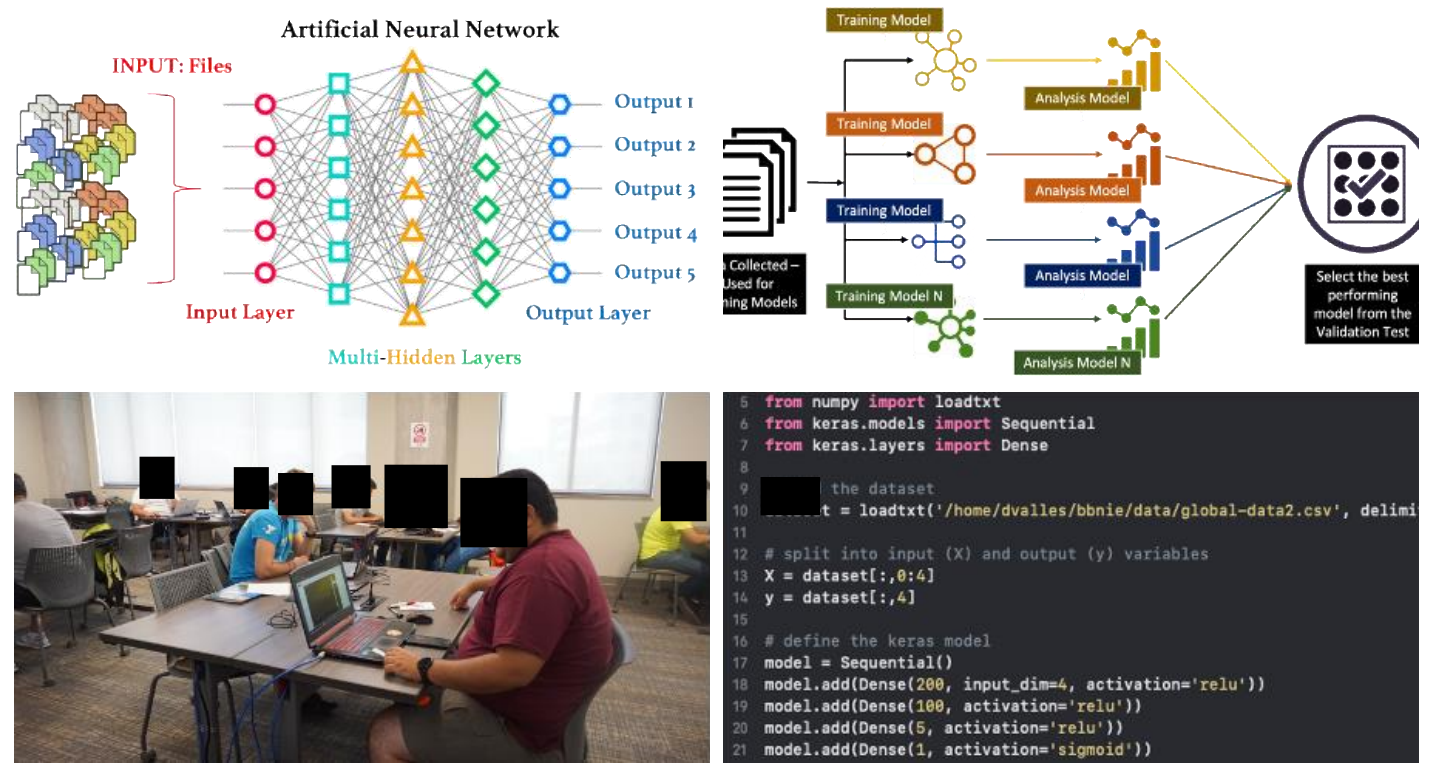

Figure A.5. Photographs of Engineering Activity 5 